\title{
Effect of Controlled Hypertension on Hemostasis Before Closure of the Operative Incision in Patients Undergoing Vertical Partial Laryngectomy: A Randomized Controlled Study
}

\section{Danyun Fu}

Fudan University Eye Ear Nose and Throat Hospital

\section{Yiting Pan}

Fudan University Eye Ear Nose and Throat Hospital

Qing Fan ( $\square$ fanqing@eentanesthesia.com )

Eye \& ENT Hospital of Fudan University

\section{Ying Guo}

Fudan University Eye Ear Nose and Throat Hospital

\section{Ming Zhang}

Fudan University Eye Ear Nose and Throat Hospital

\section{Research article}

Keywords: volume of drainage, hypertension, vertical partial laryngectomy, coughing, nausea and vomiting

Posted Date: October 5th, 2020

DOI: https://doi.org/10.21203/rs.3.rs-45636/v1

License: (c) (i) This work is licensed under a Creative Commons Attribution 4.0 International License.

Read Full License 


\section{Abstract}

Background: To evaluate the efficacy of controlled hypertension on hemostasis before closure of the operative incision in vertical partial laryngectomy (VPL).

Methods: Seventy males scheduled for VPL were randomly assigned to Group H who received either ephedrine or phenylephrine to maintain a mean arterial pressure (MAP) of $90-110 \mathrm{mmHg} 10 \mathrm{~min}$ before the operative incision closure or to Group N who received normal saline. The primary endpoint was the volume of drainage after surgery. Heart rate, MAP, surgical field bleeding score before and after drug administration, the amount of liquid administered, operation and recovery durations were recorded. Heart rate, MAP, incisional bleeding score, coughing score, nausea and vomiting score, and frequency of bloody sputum aspiration were also recorded when the patient left the postanesthesia care unit and at 6 and 24 hours postoperatively.

Results: The intraoperative bleeding score of the surgical field was significantly higher in patients receiving vasopressor. The volume of drainage in control group was more than that in Group $\mathrm{H}$ at different time points after surgery. Compared with Group N, Group H had a significantly lower incisional bleeding score and frequency of bloody sputum aspiration at all time-points after surgery, and a significantly lower nausea and vomiting score at 6 and 24 hours postoperatively.

Conclusion: Controlled hypertension (MAP 90-110 mmHg) before wound closure may be used as an alternative way to detect intraoperative bleeding points in patients undergoing VPL, which also decreased the postoperative volume of drainage and the incidences of nausea and vomiting.

Trial registration: The China Clinical Research Information Service (registration number, ChiCTR1900026097). Registered 21 September 2019, http://www.chictr.org.cn/showproj.aspx? proj $=37850$

\section{Background}

Laryngeal cancer remains the fifth to sixth most common tumour in humans, accounting for $2.5 \%$ of all tumours in males [1]. Vertical partial laryngectomy (VPL) is the most common type of open laryngectomy in our hospital, aiming to preserve a functional larynx [2]. Postoperative hemorrhage is a relatively rare but potentially life threatening and unpredictable complication in patients undergoing VPL, usually occurring within 24 hours of operation [3]. Flow of incisional blood into the lower respiratory tract may cause serious coughing. A rapidly expanding hematoma may even lead to compression and potentially lethal obstruction of the airway. Furthermore, rapid bleeding may result severe hemorrhage shock. Therefore, it is imperative to meticulously check for the likelihood of bleeding vessels, especially veins that could have been left open at the end of the surgery.

Occasionally, the surgeon is uncertain about hemostasis and thus ask the anesthesiologist to perform a Valsalva maneuver or Trendelenburg position to identify bleeding points. However, the efficacy of these 
two methods are limited and did not reduce the risk of posoperative bleeding [4]. The use of vasopressors to induce hypertension is one part of the triple-H therapy used to reverse neurological symptoms in patients with aneurysmal subarachnoid hemorrhage [5]. However, the strategy of controlled hypertension has rarely been applied in other aspects. In our preliminary study, we anesthesiologist performed a controlled hypertension before incisional closure in VPL, which increased cervical venous pressure, causes bleeding and stresses possible bleeding points, enabled the surgeons to stop bleeding more effectively.

However, the preliminary study had a small sample size, and more patients need to be continuously included to strengthen the reliability of the study results. Moreover, it remains unknown whether such controlled hypertension is safe, and whether an improved hemostatic effect reduces the incidence of postoperative complications such as coughing, nausea, and vomiting.

The present study aimed to evaluate whether the use of controlled hypertension facilitated in hemostasis in VPL, and its influence on postoperative bleeding, volume of drainage, coughing, nausea and vomiting, and the frequency of bloody sputum aspiration.

\section{Methods}

\subsection{Study Participants}

This prospective and randomized controlled trial was registered with the China Clinical Research Information Service (registration number, ChiCTR1900026097). The trial protocol was approved on August 8, 2019 by the Ethics Committee of the Eye \& ENT Hospital, Fudan University (approval no. 2019057). Written informed consent was obtained from all patients before randomization and surgery. There were 70 men eligible for participation who were 35-65 years of age, had a BMl of $19-30 \mathrm{~kg} / \mathrm{m}^{2}$ and an American Society of Anesthesiologists (ASA) grade I or II were scheduled to undergo elective VPL (oncology classification: cT1NM-cT3NM) between September 2019 and June 2020. Patients were excluded if they had abnormal coagulation function, history of cardiopulmonary and cerebrovascular diseases, history of head and neck chemoradiotherapy and/or neck surgery, history of allergy to anesthetic drugs, or were enrolled in another study. The operations were performed using a similar technique by otolaryngologist who had each performed more than 30 times of VPL procedures.

\subsection{Study Interventions}

On arrival in the operating room, the patients received routine monitoring, including electrocardiography, non-invasive blood pressure, and pulse oxygen saturation. Subsequently, a 20-G intravenous cannula was inserted and patients were intravenously injected with sufentanil $0.2 \mu \mathrm{g} / \mathrm{kg}$, propofol $2-3 \mathrm{mg} / \mathrm{kg}$, rocuronium $0.6 \mathrm{mg} / \mathrm{kg}$, and remifentanil $0.5-1 \mu \mathrm{g} / \mathrm{kg}$ for the induction of general anesthesia, followed by orotracheal intubation. Intraoperatively, anesthesia was maintained with $2.5-3.5 \%$ sevoflurane in oxygen at an age-adjusted minimum alveolar concentration of $0.9-1.1$, remifentanil $0.1 \mu \mathrm{g} / \mathrm{kg} / \mathrm{min}$ (dosage 
adjusted in accordance with blood pressure and heart rate (HR)), dexmedetomidine $1 \mu \mathrm{g} / \mathrm{kg}$, and a total intraoperative dosage of sufentanil of $0.5 \mu \mathrm{g} / \mathrm{kg}$. Initial respiratory parameters were set as follows: pressure-controlled ventilation; inspiratory pressure, $10-15 \mathrm{~cm} \mathrm{H}_{2} \mathrm{O}$; respiratory frequency, $10 \mathrm{bpm}$; ratio of inspiratory time to expiratory time, 1:1.5-1:2.0; fraction of inspired oxygen, 0.5 . The parameters were adjusted to maintain a tidal volume of $7-8 \mathrm{ml} / \mathrm{kg}$ and an end-tidal $\mathrm{CO}_{2}$ pressure of $35-45 \mathrm{mmHg}$. Body temperature was monitored and maintained at $36.0-37.0^{\circ} \mathrm{C}$ throughout surgery.

At the end of surgery, $2 \%$ lidocaine hydrochloride $(1.5 \mathrm{mg} / \mathrm{kg})$ was dripped into the balloon-type silica gel tracheostomy tube (TOT) before placement upon completion of surgery. The balloon pressure of the endotracheal TOT was consistently $40 \mathrm{~cm} \mathrm{H}_{2} \mathrm{O}$ (manometer, Covidien, Germany). Postoperatively, residual muscle relaxants were antagonized with neostigmine $0.02 \mathrm{mg} / \mathrm{kg}$ and atropine $0.01 \mathrm{mg} / \mathrm{kg}$. Patients received postoperative self-controlled analgesia with sufentanil $0.04 \mu \mathrm{g} / \mathrm{kg} / \mathrm{h}$, dexmedetomidine $0.08 \mu \mathrm{g} / \mathrm{kg} / \mathrm{h}$, and granisetron $2 \mu \mathrm{g} / \mathrm{kg} / \mathrm{h}$.

Patients were randomly assigned to receive vasopressor or normal saline by computer-generated allocations in concealed opaque envelopes into one of the two groups 35 patients each. The random allocation sequence was generated by an investigator not involved in the study. In the controlled hypertension group (Group H), the patients received intravenous administrations of ephedrine $6 \mathrm{mg}$ (maximum dose not exceeding $30 \mathrm{mg}$ ) intermittently or continuous infusion of phenylephrine $10 \mathrm{~min}$ before the closure of the operative incision $\left(T_{a}\right)$. The target mean arterial pressure (MAP) was maintained between 90 and $110 \mathrm{mmHg}$. Ephedrine was administered when the HR was $<60 \mathrm{bpm}$, while phenylephrine was administered when the HR was $>60 \mathrm{bpm}$. In the normal saline group (Group N), the patients were intravenously injected with a placebo comprising normal saline $0.1 \mathrm{ml} / \mathrm{kg}$ at $\mathrm{T}_{\mathrm{a}}$.

\subsection{Study Outcomes}

The HR and MAP were recorded at the time of arrival in the operating room (the mean of three measurements was used as the baseline value), $T_{a}$, after drug administration $\left(T_{b}\right)$ (at the time of the highest MAP), at the time of leaving the postanesthesia care unit (PACU) $\left(T_{1}\right), 6$ hours postoperatively $\left(T_{2}\right)$, and 24 hours postoperatively $\left(T_{3}\right)$. The bleeding score of the surgical field was recorded at $T_{a}$ and $T_{b}$. Operation duration (defined as the time from the creation of the skin incision to the suturing of the cervical wound) and recovery duration (defined as the time from the end of surgery to the ability to open the eyes in response to calling) were also recorded. The amount of fluid administered and the cumulative dosage of vasoactive drugs (ephedrine and phenylephrine) were also recorded.

The primary endpoint of the study was the drainage $(\mathrm{ml})$ (defined as the volume aspirated from the suction pipe above the balloon of the TOT plus the negative pressure drainage). The secondary outcomes were intraoperative bleeding score, postoperative incisional bleeding score, coughing score, nausea and vomiting score, and frequency of bloody sputum aspiration were recorded at $T_{1}, T_{2}$, and $T_{3}$. Any new 
cardiovascular and cerebrovascular complications, hematoma and reoperation that occurred were also recorded.

The intraoperative bleeding score of the surgical field was assessed based on a four-point scale where 0 indicated no bleeding (excellent operative field), 1 indicated minimal bleeding (sporadic suction), 2 indicated diffuse bleeding (repeated suction), and 3 indicated considerable bleeding (continuous suction) [6]. The degree of postoperative incisional bleeding was recorded using a system in which nine equal squares were drawn on the gauze pad under the TOT, and 0 indicated no blood or almost no blood on the pad, 1 indicated that only one square was soaked with blood, and 9 indicated that all nine squares were soaked with blood [7]. Nausea and vomiting was scored as 0 , no nausea and vomiting; 1 , only nausea, no vomiting; 2 , transient vomiting with nausea; 3 , vomiting requiring treatment; 4 , uncontrollable vomiting [8]. The number of coughs per patient was continuously monitored for 15 min postoperatively; coughing was assessed using a four-point scale as 1 , no coughing; 2 , minimal coughing (one to two times); 3 , moderate coughing (three to four times), or 4 , severe coughing ( $\geq$ five times) [9]. The intraoperative bleeding score was assessed by the surgeon who was blinded to the study groups. Each postoperative score was evaluated by a nurse anesthetist who was blinded to the experimental groups.

\subsection{Statistical Analysis}

The number of patients in each group was based on our preliminary study and a statistical power of $80 \%$ and an estimate of a $30 \%$ improvement in volume of drainage, in the first 24 hours postoperatively in Group $\mathrm{N}$ compared with Group $\mathrm{H}$ with a $5 \%$ significance level. Statistical analyses were performed using SPSS 19.0 (Chicago, IL, USA). MAP and HR were expressed as mean (standard deviation, SD) and the significance was examined by two-way analysis of variance after repeated measures and results at the same time point were compared using post hoc Bonferroni test. Age, body weight, BMI, duration of surgery, recovery time, temperature and fluid infusion were analyzed using Student's t-test. The coughing score and nausea and vomiting score were analyzed using the chi-square test. The volume of drainage, degree of intraoperative bleeding, postoperative incisional bleeding and frequency of sputum aspiration

were presented as median (interquartile range, IQR) and were compared using the Kruskal-Wallis test. $P<$ 0.05 was considered statistically significant.

\section{Results}

\subsection{Study Population}

A total of 70 patients were screened for eligibility, and 69 patients were ultimately enrolled and analyzed (Fig. 1). One patient in Group $\mathrm{H}$ did not undergo surgery and did not receive the study intervention.

\subsection{Baseline Patient Characteristics}


There were no significant differences between groups regarding age, weight, BMI, ASA grade, operation and recovery durations, end-tidal $\mathrm{CO}_{2}$, body temperature (Table $1, P>0.05$ ). The volumes of fluid administered were not different between the two study groups (Table $1, P>0.05$ ). The dosage of vasoactive drugs in Group H were recorded in Table 1.

Table 1

Baseline patient characteristics, operation and recovery durations

\begin{tabular}{|c|c|c|}
\hline & $\begin{array}{l}\text { Group N } \\
(n=35)\end{array}$ & $\begin{array}{l}\text { Group H } \\
(n=34)\end{array}$ \\
\hline Ages (years) & $55.57 \pm 6.57$ & $53.06 \pm 8.74$ \\
\hline Weight (kg) & $72.79 \pm 10.11$ & $68.38 \pm 9.25$ \\
\hline BMI & $24.71 \pm 2.99$ & $23.37 \pm 2.12$ \\
\hline 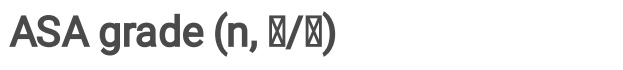 & $11 / 24$ & $9 / 25$ \\
\hline Duration of Operation (minutes) & $107 \pm 29$ & $105 \pm 31$ \\
\hline Duration of recovery (minutes) & $39 \pm 7$ & $42 \pm 8$ \\
\hline Body temperature $\left({ }^{\circ} \mathrm{C}\right)$ & $36.8 \pm 0.4$ & $36.7 \pm 0.3$ \\
\hline Fluid infusion (ml) & $1320 \pm 240$ & $1280 \pm 220$ \\
\hline \multicolumn{3}{|l|}{ Vasoactive drugs } \\
\hline Ephedrine (mg) & 0 & $15 \pm 3$ \\
\hline Phenylephrine (ug) & 0 & $250 \pm 48$ \\
\hline
\end{tabular}

\subsection{Hemodynamic Parameters}

The MAP of patients in Group $\mathrm{H}$ was significantly higher than that of patients in Group $\mathrm{N}$ at $\mathrm{T}_{\mathrm{b}}$ due to the administration of vasopressors (Table 2, $P<0.05$ ). Compared with Group N, Group H tended to have a higher HR; however, this difference was not statistically significant (Table 2, $P>0.05$ ). 
Table 2

Hemodynamic parameters

\begin{tabular}{|c|c|c|c|c|c|c|c|}
\hline & Group & Baseline & $\mathrm{T}_{\mathrm{a}}$ & $T_{b}$ & $\mathrm{~T}_{1}$ & $\mathrm{~T}_{2}$ & $T_{3}$ \\
\hline \multirow{2}{*}{$\begin{array}{l}\text { MAP } \\
(\mathrm{mmHg})\end{array}$} & Group N $(n=35)$ & $101 \pm 12$ & $73 \pm 9$ & $74 \pm 11$ & $104 \pm 13$ & $99 \pm 4$ & $101 \pm 6$ \\
\hline & Group H $(n=34)$ & $101 \pm 13$ & $68 \pm 4$ & $98 \pm 6^{*}$ & $105 \pm 20$ & $93 \pm 9$ & $95 \pm 7$ \\
\hline \multirow{2}{*}{$\begin{array}{l}\text { HR } \\
\text { (bpm) }\end{array}$} & Group N $(n=35)$ & $77 \pm 7$ & $65 \pm 5$ & $68 \pm 6$ & $82 \pm 10$ & $82 \pm 10$ & $80 \pm 9$ \\
\hline & Group $\mathrm{H}(\mathrm{n}=34)$ & $75 \pm 9$ & $68 \pm 8$ & $75 \pm 10$ & $78 \pm 12$ & $76 \pm 10$ & $78 \pm 8$ \\
\hline \multicolumn{8}{|c|}{$\begin{array}{l}\text { Values are expressed as mean }(\mathrm{SD}) \text {. MAP }=\text { mean arterial pressure, } \mathrm{HR}=\text { heart rate, Group } \mathrm{N}=\text { normal } \\
\text { saline group, Group } \mathrm{H}=\text { controlled hypertension group, } \mathrm{T}_{a}=10 \mathrm{~min} \text { before the closure of the operative } \\
\text { incision, } \mathrm{T}_{\mathrm{b}}=\text { after drug administration, } \mathrm{T}_{1}=\text { when leaving PACU, } \mathrm{T}_{2}=6 \text { hours postoperatively, } \mathrm{T}_{3}=24 \\
\text { hours postoperatively. }{ }^{*} P<0.001 \text { compared with Group } \mathrm{N} \text {. }\end{array}$} \\
\hline
\end{tabular}

\subsection{Bleeding Score Of The Surgical Field}

The intraoperative bleeding score of the surgical field was similar in the two groups at $\mathrm{T}_{\mathrm{a}}$. Unsurprisingly, compared with Group N (1 (1-1)), the bleeding score of the surgical field was significantly greater in Group $\mathrm{H}$ after vasopressor administration (2 (2-3), $P<0.05)$ (Fig. 2).

\subsection{Changes in postoperative drainage, incisional bleeding score, coughing, nausea and vomiting score and bloody sputum aspiration}

In contrast, Group $\mathrm{H}$ had a significantly lesser volume of drainage accumulated from the suction pipe above the TOT balloon plus negative pressure drainage than Group $N$ at $T_{1}, T_{2}$, and $T_{3}$ (Fig. 3. $P<0.05$, respectively). Similarly, lower incisional bleeding scores were seen in Group $\mathrm{H}$ than Group $\mathrm{N}$ at $\mathrm{T}_{1}, \mathrm{~T}_{2}$, and $\mathrm{T}_{3}$ (Table 3, $P<0.05$, respectively). 
Table 3

Coughing score, nausea and vomiting score, and frequency of bloody sputum aspiration

\begin{tabular}{|c|c|c|c|c|c|}
\hline & Group & $\mathbf{n}$ & $\mathrm{T}_{1}$ & $\mathrm{~T}_{2}$ & $\mathrm{~T}_{3}$ \\
\hline \multirow[t]{2}{*}{ Incisional bleeding scores } & $\mathrm{N}$ & 35 & $4(2-7)$ & $8(7-9)$ & $11(9-14)$ \\
\hline & $\mathrm{H}$ & 34 & $2(1-4)^{\star}$ & $5(2.5-6)^{\star \star \star}$ & $5(3.5-6)^{\star \star \star}$ \\
\hline \multirow[t]{2}{*}{ Coughing score $(n, 0 / 1 / 2 / 3)$} & $\mathrm{N}$ & 35 & $22 / 8 / 3 / 2$ & $16 / 13 / 4 / 2$ & $17 / 12 / 4 / 2$ \\
\hline & $\mathrm{H}$ & 34 & $23 / 7 / 3 / 1$ & $15 / 14 / 3 / 2$ & $16 / 13 / 3 / 2$ \\
\hline \multirow{2}{*}{$\begin{array}{l}\text { Nausea and vomiting score } \\
(\mathrm{n}, 0 / 1 / 2 / 3 / 4)\end{array}$} & $\mathrm{N}$ & 35 & $33 / 2 / 0 / 0 / 0$ & $21 / 8 / 4 / 2 / 0$ & $19 / 7 / 6 / 3 / 0$ \\
\hline & $\mathrm{H}$ & 34 & $32 / 2 / 0 / 0 / 0$ & $29 / 3 / 2 / 0 / 0^{*}$ & $27 / 4 / 3 / 0 / 0^{*}$ \\
\hline \multirow[t]{2}{*}{ Frequency of bloody sputum aspiration } & $\mathrm{N}$ & 35 & $1(0-2)$ & $3(2-4)$ & $3(2-4)$ \\
\hline & $\mathrm{H}$ & 34 & $0(0-1)^{*}$ & $2(1-3)^{*}$ & $2(2-3)^{*}$ \\
\hline \multicolumn{6}{|c|}{$\begin{array}{l}\text { Coughing score and nausea and vomiting score are expressed as the number of patients, while other } \\
\text { values are expressed as median (IQR). Group } N=\text { normal saline group, Group } H=\text { controlled } \\
\text { hypertension group, } \mathrm{T}_{1}=\text { when leaving PACU, } \mathrm{T}_{2}=6 \text { hours postoperatively, } \mathrm{T}_{3}=24 \text { hours }\end{array}$} \\
\hline
\end{tabular}

No significant differences were seen in coughing scores were between Group $\mathrm{H}$ and Group $\mathrm{N}$ at three postoperative time-points (Table $3, P>0.05$ ). There was no significant difference in the nausea and vomiting score between the two groups at $\mathrm{T}_{1}$; however, the score was significantly lower in Group $\mathrm{H}$ than Group $\mathrm{N}$ at $\mathrm{T}_{2}$ and $\mathrm{T}_{3}$ (Table $3, P<0.05$ ). The frequency of bloody sputum aspiration was significantly lower in Group $\mathrm{H}$ than Group $\mathrm{N}$ at $\mathrm{T}_{1}, \mathrm{~T}_{2}$, and $\mathrm{T}_{3}$ (Table $3, P<0.05$, respectively).

\subsection{Adverse Events}

There is no significant difference in the postoperative complications between two study groups. No cardiovascular and cerebrovascular complications occurred within 24 hours postoperatively in either group. Only 1 patient in the group without the controlled hypertension was required reoperation to control hemorrhage at 3 hours after sugery, whereas none of the patients experienced postoperative hemorrhage or reoperation in the controlled hypertension group.

\section{Discussion}

Postoperative hemorrhage is one of the most severe postoperative complications in head and neck surgery. VPL is the most common type of open laryngectomy in our hospital [2], which has a higher incidence of postoperative bleeding than total laryngectomy, mainly due to the following reasons: (1) 
Ineffective ligation of the superior laryngeal artery and its branches; (2) Preserved thyroid cartilage wing cover the thyroid cartilage stump, therefore preserved abundant small mucosal capillaries may rebleed due to postoperative hypertension caused by stress responses to pain; (3) Preservation of thyroid cartilage formed of a cavity in the throat, which is unable to provide compression hemostasis on the open cervical tissue; (4) Coughing caused by TOT placement. Once hemorrhage occurs, patients may be required urgent surgical intervention, including reopening of the wounds and hemostatic exploration $[3$, 10]. Furthermore, blood flowing into the lower respiratory tract may lead to coughing and even respiratory tract obstruction, meanwhile the accidental swallowing of imperceptible amounts of extravasated blood may result in nausea and vomiting [11]. In addition, massive hemorrhage may result in hemorrhagic shock or even death. Therefore, effective hemostasis before incisional closure is important to prevent postoperative bleeding.

Valsalva maneuver and Trendelenburg positioning performed by anesthesiologists are two techniques to help identify potential bleeding sites, through increasing internal jugular vein pressure by causing backflow of venous. However, there are some complications associated with Valsalva maneuver, such as potential pneumothorax and bradycardia, and Trendelenburg positioning has a potential risk of increase in intracranial pressure. The studies reported that Valsalva maneuver and Trendelenburg's positioning should not continue for longer than 1 minute $[12,13]$. Thus, there is an urgent need for developing novel strageties for intraoperative bleeding points detection. In the present study, controlled hypertension before wound closure significantly increased the bleeding degree in the surgical field, which was beneficial for surgeons to dectect bleeding point, and thus reducing postoperative incisional bleeding.

Triple-H therapy, including the induction of hypertension, was originally used in patients with aneurysmal subarachnoid hemorrhage after aneurysm clipping; this therapy relieves cerebral vasospasm, restores ischemic cerebral blood flow, alleviates ischemic and hypoxic injuries, and thus reverses ischemic neurological deficits [5]. However, raising of the blood pressure is rarely applied in other aspects. In the present study, the MAP was raised to $90-110 \mathrm{mmHg}$ by administering vasopressors from $T_{a}$ to $T_{b}$ to evaluate whether controlled hypertension helped surgeons attain better hemostasis.

The target MAP value (90-110 $\mathrm{mmHg}$ ) was based on the blood pressure range at the time of leaving the PACU in a preliminary experiment involving 10 patients undergoing VPL. We provide the data of preliminary study as supplementary data. No substantial cardiovascular and cerebrovascular complications were caused by the transient raising of the MAP in our study. However, patients were excluded if they had a history of cardiopulmonary and cerebrovascular diseases, so we cannot claim that increasing MAP to $90-110 \mathrm{mmHg}$ may not cause complications in these patients. Furthermore, the choice of vasoactive drug depends on the patient's HR. Ephedrine is a vasopressor that has $\alpha_{1}, \beta_{1}$, and $\beta_{2}$ effects mediated by the release of endogenous catecholamines from adrenergic nerve endings, resulting in increases in both HR and MAP [14]. Thus, ephedrine was used when the HR was $<80 \mathrm{bpm}$.

Phenylephrine is a selective $a_{1}$-adrenergic agonist that results in increased blood pressure and has a negative effect on HR, and so it was used when the HR was $>80 \mathrm{bpm}[14]$. 
As expected, the intraoperative bleeding score of the surgical field was significantly higher in Group $\mathrm{H}$ than in Group N in our study. However, the postoperative drainage and incisional bleeding score were significantly lesser in Group $\mathrm{H}$ than in Group $\mathrm{N}$ at $\mathrm{T}_{1}, \mathrm{~T}_{2}$, and $\mathrm{T}_{3}$, which suggests that the use of controlled hypertension before wound closure is helpful for hemostasis and thus reducing the postoperative incisional bleeding. Furthermore, nausea and vomiting was alleviated in Group H compared with Group N, which may be due to lesser extravasated blood was accidentally swallowed in patients receiving controlled hypertension.

Among the risk factors leading to postoperative hemorrhage in VPL surgery, postoperative hypertension, coughing, the duration of operation, and the experience of surgeon are the most likely ones. While, hypertension was ragarded as the most risky one. Therefore, patients received sufficient analgesia intraand postoperatively to attenuate the postoperative hemodynamic changes caused by wound pain. Also, there is always a cough due to TOT placement after surgery, which elevates venous pressure and increases the risk of bleeding. $2 \%$ lidocaine hydrochloride was dripped into the endotracheal TOT to avoid bias caused by coughing in our study. As expected, there was no significant difference between the two groups in the coughing score after using $2 \%$ lidocaine hydrochloride. Excessive cuff pressure may cause ischemic injury of the tracheal mucosa, while inadequate cuff pressure may increase the risk of aspiration of blood and gastric contents. Furthermore, to avoid bias caused by variations in surgical technique, all surgical procedures were performed by experienced otolaryngologist with more than 30 times of VPL operations. No difference was observed regarding the duration of operation. Postoperative data collection was performed by a nurse anesthetist who was blinded to the group assignments.

The present study has several limitations. Firstly, the research outcomes and complications might be affected by variations in operative techniques, as the surgery procedures were performed by different surgeons. Secondly, there may have been substantial variations in blood pressure. The optimal duration and target MAP of controlled hypertension require further investigation. Thirdly, the drug intervention could not be blinded, but the risk of bias was minimized through computer-generated randomization to ensure the concealment of study group allocations. To avoid observer bias, the validated criteria for the outcomes were assessed by the surgeon and nurse anesthetist who were blinded to the experimental groups. Fourth, the sample size was relatively small.

\section{Conclusion}

The present study shows that administering vasopressors to raise the MAP to $90-110 \mathrm{mmHg}$ before wound closure in patients undergoing VPL is beneficial in enabling surgeons to effectively detect bleeding points and thus reduce postoperative incisional bleeding. Furthermore, improved incisional bleeding reduces the incidences of nausea and vomiting, and the frequency of bloody sputum aspiration, which contribute to postoperative recovery. We suggest that the patients should routinely be given controlled hypertension during hemostasis in patients undergoing VPL and it can also be used in other surgeries which may be prone to postoperative bleeding. 


\section{Abbreviations}

VPL: vertical partial laryngectomy; ASA: American Society of Anesthesiologists; MAP: mean arterial pressure; HR: heart rate; PACU: postanesthesia care unit; TOT: balloon-type silica gel tracheostomy tube; SD: standard deviation; IQR: Interquartile range.

\section{Declarations}

\section{Ethics approval and consent to participate}

All included patients gave their written informed consent. The trial protocol was approved on August 8, 2019 by the Ethics Committee of the Eye \& ENT Hospital, Fudan University (approval no. 2019057).

\section{Consent for publication}

Not applicable.

\section{Availability of data and materials}

The datasets used and/or analyzed during the current study are available from the corresponding author on reasonable request.

\section{Competing interests}

The authors declare that they have no competing interests.

\section{Funding}

There was no funding source in this study.

\section{Authors' contributions}

Qing Fan conceived the study and participated in its design and coordination. Danyun Fu conducted the study and helped to revise the study protocol. Yiting Pan collected the data. Ying Guo performed the statistical analysis and provided advice. Ming Zhang revised the study protocol and revised the manuscript. All authors have read and approved the manuscript.

\section{Acknowledgements}

We thank Kelly Zammit, BVSc, from Liwen Bianji, Edanz Editing China (www.liwenbianji.cn/ac), for editing the English text of a draft of this manuscript.

\section{Authorship Attribution}


Qing Fan conceived the study and participated in its design and coordination. Danyun Fu conducted the study and helped to revise the study protocol. Yiting Pan collected the data. Ying Guo performed the statistical analysis and provided advice. Ming Zhang revised the study protocol and revised the manuscript. All authors have read and approved the manuscript.

\section{Conflict of Interest}

The authors declare that no competing interests exist.

\section{Clinical trial registration}

This prospective and randomized controlled trial was registered with the China Clinical Research Information Service (registration number, ChiCTR1900026097).

\section{References}

1. Thomas L, Drinnan M, Natesh B, Mehanna H, Jones T, Paleri V. Open conservation partial laryngectomy for laryngeal cancer: a systematic review of English language literature. Cancer treatment reviews. 2012;38(3):203-11.

2. Yang $Y$, Zhou J, Chen M, Fang Y, Tao L, He P, Cheng L, Wu H. A study of the association between local recurrence and surgical margins in vertical partial laryngectomy for T1 glottic squamous cell carcinoma. Acta oto-laryngologica. 2019;139(8):707-12.

3. Gallo O, Locatello LG, Larotonda G, Napoleone V, Cannavicci A. Nomograms for prediction of postoperative complications in open partial laryngeal surgery. Journal of surgical oncology. 2018;118(6):1050-7.

4. Tokac M, Dumlu EG, Bozkurt B, Ocal H, Aydin C, Yalcin A, Cakir B, Kilic M. Effect of Intraoperative Valsalva Maneuver Application on Bleeding Point Detection and Postoperative Drainage After Thyroidectomy Surgeries. International surgery. 2015;100(6):994-8.

5. Sen J, Belli A, Albon H, Morgan L, Petzold A, Kitchen N. Triple-H therapy in the management of aneurysmal subarachnoid haemorrhage. Lancet Neurol. 2003;2(10):614-21.

6. Durmus M, But AK, Dogan Z, Yucel A, Miman MC, Ersoy MO. Effect of dexmedetomidine on bleeding during tympanoplasty or septorhinoplasty. Eur J Anaesthesiol. 2007;24(5):447-53.

7. Wang Y, Lu WS, Qiao H, Zhao J, Fan Q. Use of lidocaine to prevent postoperative coughing after partial laryngectomy: comparison of three delivery methods. Drug design, development and therapy. 2019, 13:1835-41.

8. Jantunen IT, Muhonen TT, Kataja VV, Flander MK, Teerenhovi L: 5-HT3 receptor antagonists in the prophylaxis of acute vomiting induced by moderately emetogenic chemotherapy-a randomised study. European journal of cancer (Oxford, England: 1990). 1993, 29A(12):1669-72.

9. Guler G, Akin A, Tosun Z, Eskitascoglu E, Mizrak A, Boyaci A. Single-dose dexmedetomidine attenuates airway and circulatory reflexes during extubation. Acta anaesthesiologica Scandinavica. 
2005;49(8):1088-91.

10. Jones NF, Jarrahy R, Song JI, Kaufman MR, Markowitz B. Postoperative medical complications-not microsurgical complications-negatively influence the morbidity, mortality, and true costs after microsurgical reconstruction for head and neck cancer. Plastic reconstructive surgery. 2007;119(7):2053-60.

11. Hovorka J, Korttila K, Erkola O. Gastric aspiration at the end of anaesthesia does not decrease postoperative nausea and vomiting. Anaesthesia intensive care. 1990;18(1):58-61.

12. Moumoulidis I, Martinez Del Pero M, Brennan L, Jani P. Haemostasis in head and neck surgical procedures: Valsalva manoeuvre versus Trendelenburg tilt. Ann R Coll Surg Engl. 2010;92(4):292-4.

13. Ozdemir M, Makay O, Icoz G, Akyildiz M. What adds Valsalva maneuver to hemostasis after Trendelenburg's positioning during thyroid surgery? Gland surgery. 2017, 6(5):433-6.

14. Mets B. Should Norepinephrine, Rather than Phenylephrine, Be Considered the Primary Vasopressor in Anesthetic Practice? Anesthesia and analgesia. 2016, 122(5):1707-14.

\section{Figures}




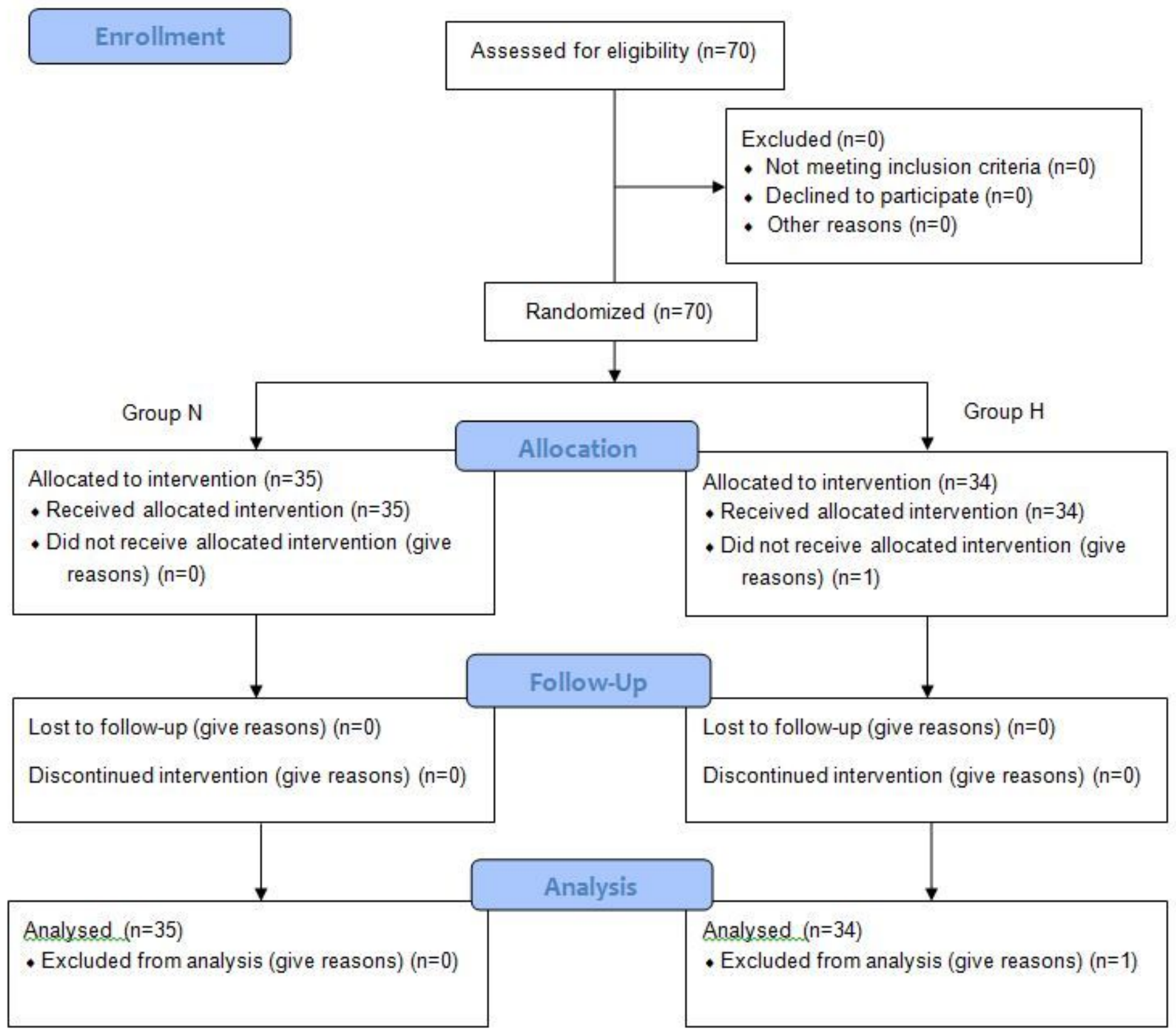

\section{Figure 1}

CONSORT flow diagram. Seventy patients scheduled to undergo VPL were randomized. One patient in Group $\mathrm{H}$ was excluded because he did not undergo surgery and did not receive the study intervention. VPL = vertical partial laryngectomy, Group N = normal saline group, Group $\mathrm{H}$ = controlled hypertension group. 


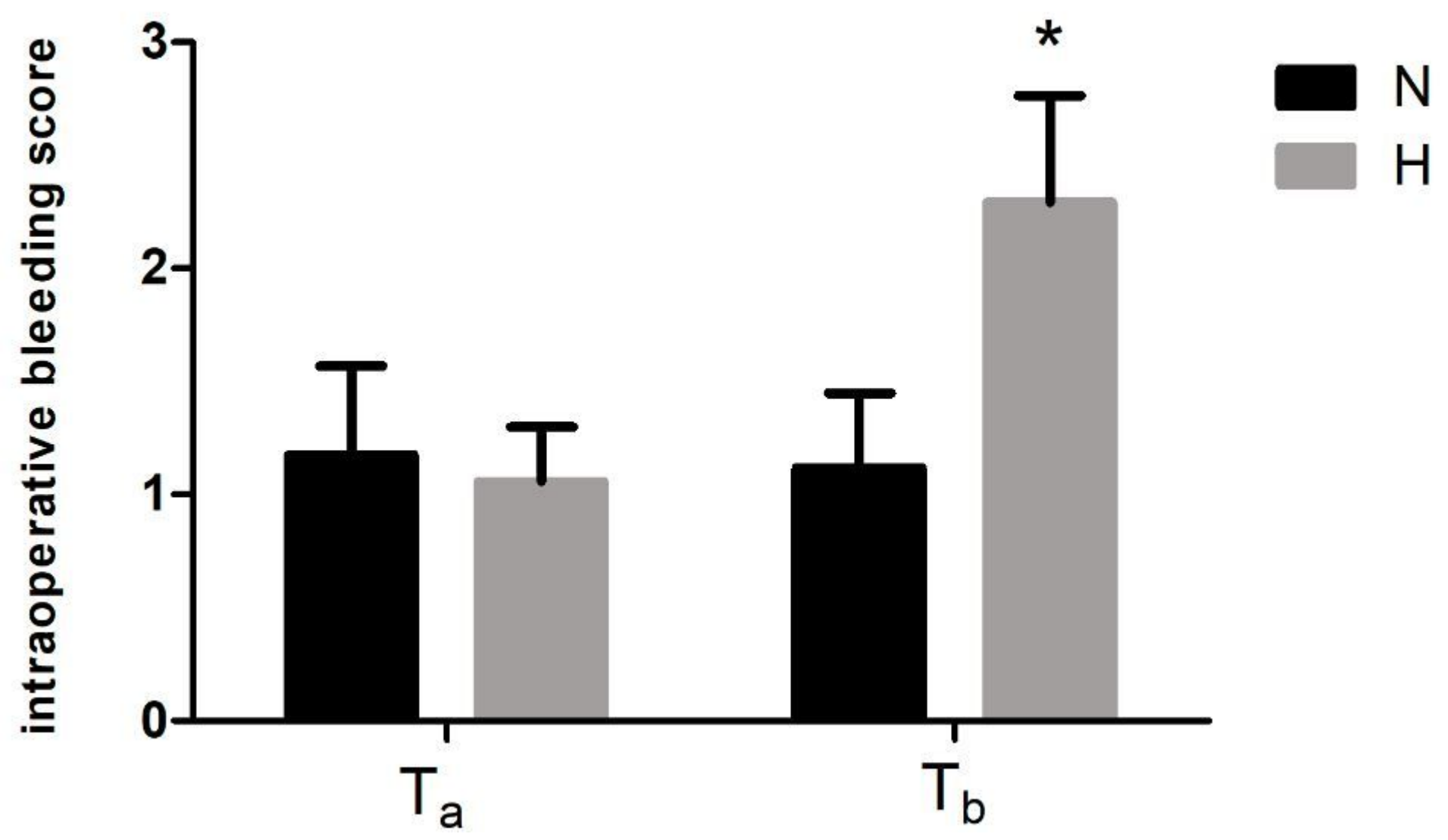

Figure 2

Intraoperative bleeding score of the surgical field. Compared with Group N, the intraoperative bleeding score of the surgical field was significantly greater in Group $\mathrm{H}$ after vasopressor administration. $\mathrm{N}=$ normal saline group, $\mathrm{H}=$ controlled hypertension group, $\mathrm{Ta}=10$ minutes before the closure of the operative incision, $\mathrm{Tb}=$ after drug administration. ${ }^{*} \mathrm{P}<0.05$ compared with Group $\mathrm{N}$.
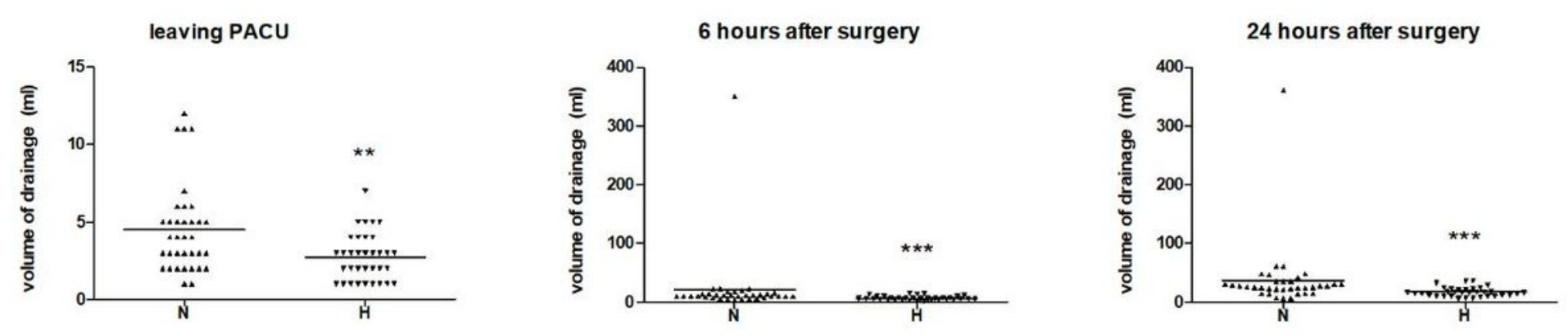

Figure 3

Volume of drainage (volume aspirated from the tracheostomy tube + volume drained from the wound via a passive closed drain) at various postoperative time-points. Compared with Group N, Group H had a significantly lesser bleeding volume at three postoperative time-points. $\mathrm{N}=$ normal saline group, $\mathrm{H}=$ controlled hypertension group. ${ }^{\star *} \mathrm{P}<0.01$ compared with Group $\mathrm{N},{ }^{\star \star *} \mathrm{P}<0.001$ compared with Group N. 


\section{Supplementary Files}

This is a list of supplementary files associated with this preprint. Click to download.

- CONSORT2010Checklist.doc

- supplementarydata.xlsx 